\title{
Usefulness of a dedicated mouthpiece for the Valsalva maneuver to visualize the hypopharynx during transoral endoscopy $\square$
}

\section{(ㄷ)(우우}

\author{
Authors \\ Minoru Kato ${ }^{1}$, Yoshito Hayashi ${ }^{1}$, Ryotaro Uema ${ }^{1}$, Hirotsugu Saiki ${ }^{1}$, Keiichi Kimura ${ }^{1}$, Takanori Inoue ${ }^{1}$, Akihiko \\ Sakatani ${ }^{1}$, Shunsuke Yoshii ${ }^{1}$, Yoshiki Tsujii ${ }^{1}$, Shinichiro Shinzaki ${ }^{1}$, Eisuke Hida ${ }^{2}$, Hideki lijima ${ }^{1}$, Tetsuo Takehara ${ }^{1}$
}

Institutions

1 Department of Gastroenterology and Hepatology, Osaka University Graduate School of Medicine, Suita, Japan

2 Department of Biostatics and Data Science, Osaka University Graduate School of Medicine, Suita, Japan

submitted 6.9.2019

accepted after revision $\quad 2.12 .2019$

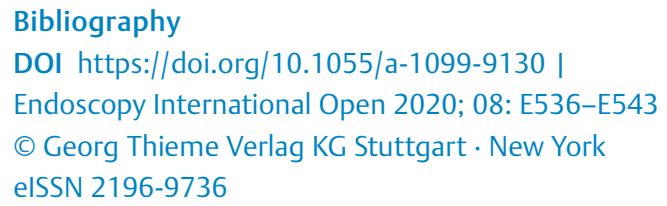

\section{ABSTRACT}

Background and study aims Patients with esophageal squamous cell carcinoma (SCC) are at high risk of develop- ing second primary SCCs in the hypopharynx. However, such second primary tumors are difficult to observe because of lumen closure. The Valsalva maneuver using a dedicated mouthpiece is a promising technique to visualize the hypopharynx during transoral endoscopy. In the current study, we investigated the utility of this method.

Patients and methods The current study was a randomized, controlled, crossover trial. Patients with esophageal SCC were randomly assigned first to undergo pharyngeal observation using the dedicated mouthpiece followed by observation using a conventional mouthpiece, or vice versa. The primary endpoint was complete visualization of the hypopharynx, which was assessed blindly by three external evaluators.

Results A total of 68 pharyngeal examinations were analyzed - 34 with the dedicated mouthpiece and 34 with a conventional mouthpiece. Complete visualization was achieved in $68 \%$ of the examinations (23/34) using the dedicated mouthpiece, whereas none of the examinations using the conventional mouthpiece achieved complete visualization of the hypopharynx. Observation scores of the oropharynx were not significantly different between both types of examination $(P=0.50)$. No serious adverse events (AEs) occurred.

Conclusions Endoscopic view of the hypopharynx was markedly improved by the Valsalva maneuver using the dedicated mouthpiece, with no serious AEs. This procedure should be included in the endoscopic examinations for the patients with esophageal SCCs.

\section{Introduction}

Multiple squamous cell carcinomas (SCCs) frequently arise in the esophagus and pharynx, which has been recognized as the field cancerization phenomenon [1,2]. Patients with esophageal SCCs, therefore, require careful monitoring of the pharynx to guarantee early detection of pharyngeal SCCs, which may increase the possibility of minimally invasive treatment, including endoscopic resection [3-8]. Narrow band imaging (NBI) has en- abled early detection of superficial SCC, not only in the esophagus but also in the pharynx [9-14]. However, due to closure of the lumen, some pharyngeal subsites, such as the hypophar$y n x$, are difficult to observe. The hypopharynx consists of the pyriform sinuses, postcricoid area, and posterior wall. Cancers in this hidden area are sometimes detected at an advanced stage, even in patients who are subject to periodic endoscopic examination [15].

The Valsalva maneuver has been used to expose the hypopharyngeal space during nasopharyngoscopy [16]. However, 

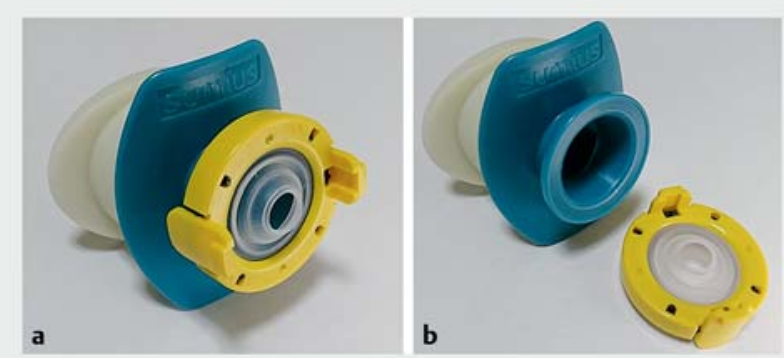

- Fig. 1 Dedicated mouthpiece for performing the Valsalva maneuver (Valsamouth; Sumitomo Bakelite, Tokyo, Japan). a The degassing prevention valve (yellow part) is attached to the mouthpiece (blue part). b The yellow valve is detachable. After pharyngeal observation, we detach this valve and start endoscopic examination of the upper gastrointestinal tract.

to detect superficial pharyngeal cancer exhibiting only minute changes in the mucosa, high-resolution transoral endoscopy is desirable [17]. In this context, a dedicated mouthpiece for performing the Valsalva maneuver during transoral endoscopy has recently been launched in Japan: the Valsamouth (Sumitomo Bakelite, Tokyo, Japan; Fig.1a) [18], on which a valve seals the gap between the endoscope and mouthpiece, preventing air leakage while the patient exhales. After pharyngeal observation, the valve can be detached ( $\triangleright$ Fig. 1b), allowing the device to be used as a conventional mouthpiece to examine the upper gastrointestinal tract. Using this novel dedicated mouthpiece, the Valsalva maneuver during transoral endoscopy is a promising diagnostic procedure to screen pharyngeal SCCs in patients with esophageal SCCs. However, no prospective studies have yet evaluated the utility of this new mouthpiece.

The aim of the current study was to investigate whether the dedicated mouthpiece improves the visibility of the hypopharynx compared with a conventional mouthpiece.

\section{Patients and methods}

\section{Study design}

This was a randomized controlled crossover trial performed at Osaka University Hospital comparing the rate of complete visualization of the hypopharynx during transoral endoscopy between examinations using the dedicated mouthpiece and those using a conventional mouthpiece. The study was designed according to the Consolidated Standards of Reporting Trials 2010 Statement. The study protocol was approved by the Institutional Review Board of Osaka University Hospital, Japan (No. 18062, dated July 12, 2018). The study was registered on July 4, 2018 in the University Hospital Medical Network Clinical Trials Registry (UMIN000033274). Written informed consent was obtained from the all patients before enrollment.

\section{Participants}

Inclusion criteria were: (1) age $\geq 20$ years; (2) current or previous diagnosis of esophageal SCC; and (3) scheduled transoral endoscopy. Exclusion criteria were: (1) contraindications to the Valsalva maneuver (i.e., aortic stenosis, myocardial infarction within the past year, retinopathy [19]); (2) advanced head and neck (H\&N) cancer, (3) history of treatment for H\&N cancer; (4) dementia, psychiatric disorder, or auditory impairment; (5) contraindications to lidocaine or pethidine hydrochloride; and (6) inability to bite a mouthpiece because of impaired mouth opening, or dental disease.

\section{Examiners and pharyngeal observation}

The examinations were performed by three experienced gastroinestinal endoscopists (M.K., R.U., and S.Y.) who were certified by the Japan Gastroenterological Endoscopy Society and had experience of at least five pharyngeal observations using the dedicated mouthpiece prior to study commencement.

Pharyngeal observation was performed using a transoral endoscope (GIF-H290Z; Olympus Medical Systems, Tokyo, Japan) and standard video endoscope system (EVIS LUCERA ELITE; Olympus).

Prior to the procedure, local pharyngeal anesthesia was performed using $2 \%$ lidocaine viscous (Xylocaine Viscous $2 \%$; AstraZeneca, Osaka, Japan). All patients then received $25 \mathrm{mg}$ of pethidine hydrochloride intravenously. This drug reduces gag reflex and discomfort without causing the patient to lose consciousness, and it is a useful analgesic for pharyngeal observation [20]. Patients' heart rate, oxygen saturation, and blood pressure were monitored during the observation.

In each patient, pharyngeal observation was performed twice consecutively by the same examiner, first with the dedicated mouthpiece and then with the conventional mouthpiece (dedicated-to-conventional), or vice-versa (conventional-todedicated) at random. When the first observation was finished, the endoscope was removed from the mouth and the patients were given a 3-minute break before the next observation.

The examiners started the observation from the oropharynx. Specifically, they observed the uvula, the posterior wall of the oropharynx, and the epiglottic vallecula. The endoscope was then gently inserted into the hypopharynx. When the hypopharyngeal observation was performed with the dedicated mouthpiece, examiners instructed patients to perform the Valsalva maneuver ( Supplementary Video 1 ). Conversely, when the observation was performed with a conventional mouthpiece, examiners instructed patients to vocalize, which is the current standard technique to improve hypopharynx visibility using a conventional mouthpiece [20,21]. During the observation, pictures of the oropharynx and the hypopharynx were taken for later evaluation by three external evaluators. The time limit for each type of examination was set at 5 minutes. All the pharyngeal observations were performed under NBI mode. 


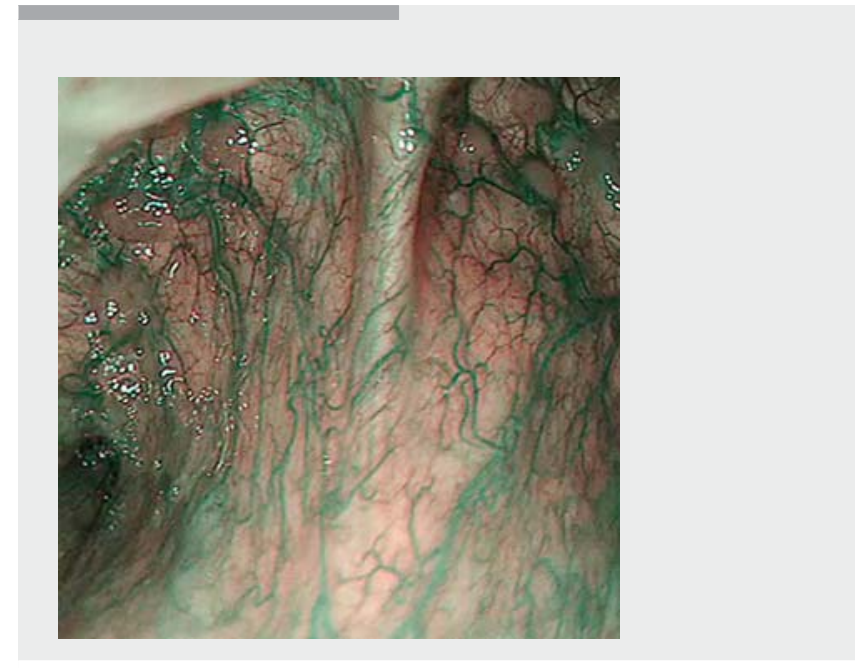

$\checkmark$ Video 1 Pharyngeal observation using the dedicated mouthpiece.

\section{Randomization}

Patients were randomly assigned to one of the following two groups at a 1:1 ratio using the random numbers generator function in Microsoft Excel 2010 (Microsoft Corporation, Redmond, Washington, United States), with no stratification: (1) dedicated-to-conventional mouthpiece group: first examination with the dedicated mouthpiece followed by subsequent examination with a conventional mouthpiece; (2) conventional-to-dedicated group: first examination with a conventional mouthpiece followed by subsequent examination with the dedicated mouthpiece. The randomization sequence was concealed to the examiners until the patients were assigned.

\section{Blinding method}

All the images of the pharyngeal examinations were collected and separated into those captured during examinations using the dedicated mouthpiece and those captured during conven- tional mouthpiece use. Patients' names and examination dates were all removed from the endoscopic images, and the case order was randomly rearranged. Three external evaluators, who had 7,15 , and 17 years of experience in gastrointestinal endoscopy, respectively, independently evaluated the processed images. All the evaluators were blinded to the type of pharyngeal observation.

\section{Scoring criteria}

Visibility of the hypopharynx was evaluated using the following scoring criteria, which were specially developed at our institution for the purpose of this study ( $\triangleright$ Fig. 2 ): Score $0=$ the pyriform sinuses, postcricoid area, and posterior wall of the hypopharynx are all invisible, and/or the captured subjects are excessively out of focus (not seen); Score 1 = both sides of the aryepiglottic fold are elevated and both sides of the pyriform sinus are visible; however, the postcricoid area and posterior wall of the hypopharynx are not visible (partially seen); Score $2=$ the postcricoid area is elevated and therefore both sides of the pyriform sinus, postcricoid area, and posterior wall of the hypopharynx are all visible (completely seen).

Before starting the actual evaluation, the scoring criteria were explained to all evaluators using representative endoscopic images. If there was any disagreement about the image scoring among the three evaluators, the score that was given most often was adopted.

\section{Outcomes}

The primary endpoint was the proportion of examinations that achieved complete visualization of the hypopharynx, defined as a score of 2 given by the external evaluators.

The secondary endpoints were: (1) total observation score of the three oropharyngeal subsites (i. e., the uvula, posterior wall of the oropharynx, and epiglottic vallecula) assessed by the external evaluators; (2) pharyngeal observation time; (3) incidence of adverse events (AEs); and (4) presence of newly detected pharyngeal neoplasm.
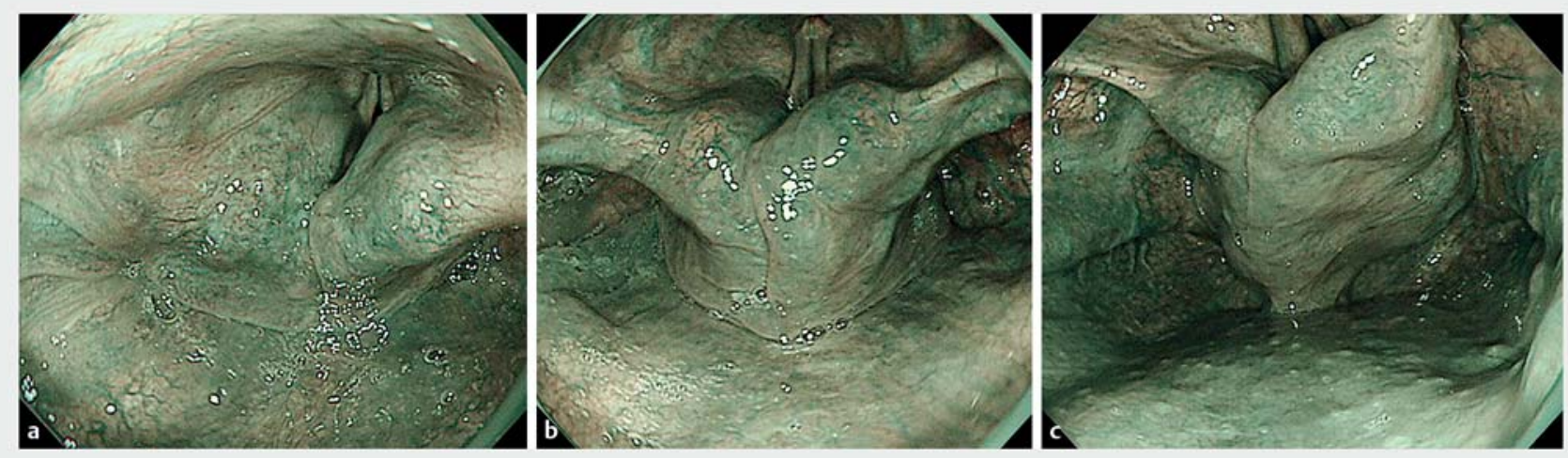

- Fig. 2 Scoring criteria for hypopharynx visibility. a Score 0: pyriform sinus, postcricoid area, and posterior wall of the hypopharynx are all invisible (not seen). b Score 1: both sides of the aryepiglottic fold are elevated and both sides of the pyriform sinus are visible; however, the postcricoid area and posterior wall of the hypopharynx are not visible (partially seen). c Score 2: postcricoid area is elevated and therefore both sides of the pyriform sinus, postcricoid area, and posterior wall of the hypopharynx are all visible (completely seen). 


\section{Assessed for eligibility $(n=46)$}

Excluded

- prior treatment for H\&N cancer $(n=4)$

- retinopathy $(n=3)$

- dementia ( $n=2)$

- psychiatric disorder $(n=1)$

- refused to participate $(n=1)$

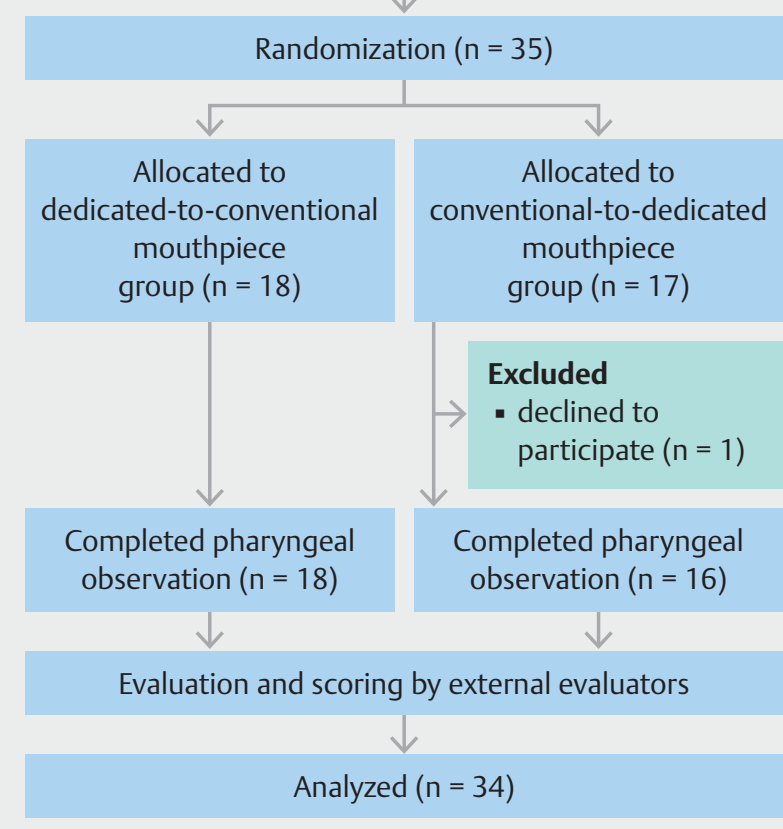

- Fig. 3 Participant flow. H\&N, head and neck.

Endoscopic images of each oropharyngeal subsite were graded as either good (1 point) or poor (0 points). Properly focused images with no excessive saliva on the captured subjects were graded as good (1 point). Otherwise, the images were graded as poor (0 points) [18,19]. Pharyngeal observation time was defined as the time between insertion of the endoscope into the oral cavity and removal of the endoscope from the oral cavity, which was measured by assistants using a stopwatch.

A post hoc analysis was performed to explore the factors associated with the incomplete visualization of the hypopharynx.

\section{Sample size calculation}

In our past clinical experience using the dedicated mouthpiece, complete visualization of the hypopharynx has been achieved in at least $50 \%$ of cases. Therefore, we hypothesized that the dedicated mouthpiece would increase the rate of complete visualization of the hypopharynx from $5 \%$ to $50 \%$. Assuming $30 \%$ discordant pairs, 30 patients were required to achieve $90 \%$
- Table 1 Patient characteristics $(n=34)$.

\begin{tabular}{|c|c|}
\hline Age, median (range), years & $72(48-87)$ \\
\hline Gender, male/female, n & $30 / 4$ \\
\hline \multicolumn{2}{|l|}{ Esophageal cancer, n } \\
\hline - Current & 28 \\
\hline - Previous & 6 \\
\hline BMI, median (range), $\mathrm{kg} / \mathrm{m}^{2}$ & $22(17-29)$ \\
\hline \multicolumn{2}{|l|}{ ECOG performance status, $n$} \\
\hline . $0-1$ & 34 \\
\hline . $2-4$ & 0 \\
\hline \multicolumn{2}{|l|}{ Drinking habit, $\mathrm{n}$} \\
\hline - Never or quit & 9 \\
\hline - Occasionally & 5 \\
\hline - More than once a week & 4 \\
\hline - Everyday & 16 \\
\hline \multicolumn{2}{|l|}{ Smoking habit, $\mathrm{n}$} \\
\hline - Never or quit & 26 \\
\hline - Yes & 8 \\
\hline \multicolumn{2}{|c|}{ Number of previous upper gastrointestinal endoscopy sessions, $n$} \\
\hline . $0-1$ & 0 \\
\hline . $2-4$ & 10 \\
\hline - $\geq 5$ & 24 \\
\hline BMI, body mass index; $\mathrm{ECOG}, \mathrm{E}$ & operative Oncology Group \\
\hline
\end{tabular}

power with a two-sided $\alpha$-level of 0.05 . We finally set the total sample size at 35 patients, with consideration for possible eligibility deviation and dropout cases. Sample size calculation was performed using $G^{*}$ Power version 3.1.9.2 (The $G^{*}$ Power Team, Düsseldorf, Germany) (Retrieved from http://www.gpower. hhu.de) [22].

\section{Statistical analysis}

To compare the two methods of pharyngeal observation in cases of continuous data, the paired $t$-test was used, while the McNemar test was used in cases of categorical data. Fisher's exact test was used to compare the categorical data between the patients who could achieve complete visualization and who could not. $P<0.05$ was considered statistically significant. Interobserver agreements between evaluators were calculated using kappa statistics. Agreement was classified as excellent with kappa values $\geq 0.8$; good with values from $<0.8$ to $\geq 0.6$; moderate for values from $<0.6$ to $\geq 0.4$; and fair for values $<0.4$. All analyses were performed using JMP Pro version 13 (SAS Institute, Cary, North Carolina, United States). 
- Table 2 Summaries of primary and secondary outcomes.

\begin{tabular}{|c|c|c|c|}
\hline & Dedicated mouthpiece & Conventional mouthpiece & $P$ value \\
\hline & $\mathrm{n}=34$ & $n=34$ & \\
\hline Rate of complete visualization of the hypopharynx, (\%) & $68(23 / 34)$ & $0(0 / 34)$ & N/A \\
\hline Observation score of the hypopharynx, mean \pm SD & $1.6 \pm 0.7$ & $0.6 \pm 0.5$ & $<0.0001^{1}$ \\
\hline Total observation score of the oropharynx, mean \pm SD & $2.6 \pm 0.7$ & $2.7 \pm 0.5$ & $0.50^{1}$ \\
\hline Pharyngeal observation time, mean \pm SD, seconds & $166 \pm 56$ & $119 \pm 59$ & $<0.0001^{1}$ \\
\hline Adverse events & & & $0.56^{2}$ \\
\hline Decrease in $\mathrm{SpO}_{2}(<90 \%)$, $\mathrm{n}$ & $1(3 \%)$ & $0(0 \%)$ & \\
\hline Tachycardia (HR >150 bpm), n & $1(3 \%)$ & $0(0 \%)$ & \\
\hline Bradycardia (HR <50 bpm), n & $0(0 \%)$ & $0(0 \%)$ & \\
\hline Increase in SBP $(>\Delta 20 \mathrm{mmHg}), \mathrm{n}$ & $1(3 \%)$ & $1(3 \%)$ & \\
\hline Decrease in $\mathrm{SBP}(>\Delta 20 \mathrm{mmHg}), \mathrm{n}$ & $0(0 \%)$ & $0(0 \%)$ & \\
\hline Vasovagal syncope, $\mathrm{n}$ & $0(0 \%)$ & $0(0 \%)$ & \\
\hline \multicolumn{4}{|c|}{$\begin{array}{l}\mathrm{N} / \mathrm{A} \text {, not available; SD, standard deviation; } \mathrm{SpO}_{2} \text {, saturation of percutaneous oxygen; } \mathrm{HR} \text {, heart rate; } \mathrm{SBP} \text {, systolic blood pressure } \\
1 \text { Paired } t \text {-test } \\
{ }^{2} \text { McNemar test }\end{array}$} \\
\hline
\end{tabular}

\section{Results}

\section{Participants}

From August 2018 to June 2019, 46 patients were assessed for eligibility. Of these, 11 were considered ineligible (four had undergone prior treatment for H\&N cancer, three had retinopathy, two had dementia, one had a psychiatric disorder, and one refused to participate). Hence, a total of 35 patients were enrolled and randomized. Eighteen patients were allocated to the dedicated-to-conventional mouthpiece group and 17 to the conventional-to-dedicated mouthpiece group. One patient allocated to the conventional-to-dedicated mouthpiece group declined to participate in the study soon after the randomization; thus, 34 patients ultimately completed the study protocol and were included in the analysis ( $\triangleright$ Fig. 3 ). Baseline characteristics of these 34 participants are shown in $>$ Table 1. Median age was 72 years (range: $48-87$ years), and 30 patients ( $88 \%$ ) were male.

\section{Study outcomes}

Primary and secondary outcomes are summarized in > Table 2. The rate of complete visualization of the hypopharynx was $68 \%$ $(23 / 34)$ in examinations that used the dedicated mouthpiece, whereas it was $0 \%(0 / 34)$ in examinations that used a conventional mouthpiece. None of the examinations using a conventional mouthpiece achieved complete visualization, so the McNemar test was not applicable to the paired results. Thus, we alternatively compared the mean ( \pm standard deviation [SD]) observation scores of the hypopharynx, which were significantly higher in the examinations using the dedicated mouthpiece ( $1.6 \pm 0.7$ points with the dedicated mouthpiece vs. $0.6 \pm 0.5$ points with the conventional mouthpiece, $P<$ $0.0001)$. The difference in mean observation score between the two examinations was 1.0 , with a two-sided $95 \%$ confidence interval of 0.7 to 1.3 . These results indicated that the hypopharynx was better visualized using the dedicated mouthpiece. Detailed scoring for all 34 patients is shown in $>$ Table 3.

Mean $( \pm S D)$ total observation scores of the oropharynx were not significantly different between the two ways of pharyngeal examination $(2.6 \pm 0.7$ points with the dedicated mouthpiece vs. $2.7 \pm 0.5$ points with the conventional mouthpiece, $P=$ $0.50)$. Average ( \pm SD) time required for pharyngeal observation was significantly longer in the examinations that used the dedicated mouthpiece (166 \pm 56 seconds vs. $119 \pm 59$ seconds; $P<$ $0.0001)$. We detected one superficial pharyngeal SCC located in the posterior wall of the hypopharynx.

There was good agreement among the three external evaluators regarding scoring of the hypopharyngeal observation, with a mean kappa value of 0.74 . The pairwise kappa values between the evaluators were $0.84,0.68$, and 0.71 .

\section{Adverse events}

No serious AEs occurred with either type of examination. None of the patients asked to stop the examination. Transient vital sign changes occurred in two patients during the examinations with the dedicated mouthpiece and in one patient during the examination with a conventional mouthpiece, but no medical intervention was required in any of these cases. Thus, incidence of AEs did not differ significantly between the two observation methods ( $\triangleright$ Table 2 ). 
- Table 3 Observation score of the hypopharynx evaluated by the three external evaluators.

\begin{tabular}{|c|c|c|c|c|c|}
\hline & & \multicolumn{3}{|c|}{ Dedicated mouthpiece $(n=34)$} & \\
\hline & & Score 0 & Score 1 & Score 2 & \\
\hline \multirow[t]{4}{*}{ Conventional mouthpiece $(n=34)$} & Score 0 & 3 & 3 & 9 & $15(44 \%)$ \\
\hline & Score 1 & 1 & 4 & 14 & $19(56 \%)$ \\
\hline & Score 2 & 0 & 0 & 0 & $0(0 \%)$ \\
\hline & & $4(12 \%)$ & $7(20 \%)$ & 23 (68\%) & 34 \\
\hline
\end{tabular}

- Table4 Association between patient characteristics and observation scores.

\begin{tabular}{|c|c|c|c|}
\hline & Score 2 & Score 0 or 1 & $P$ value ${ }^{1}$ \\
\hline & $(n=23)$ & $(n=11)$ & \\
\hline Age $\geq 72$ years $^{2}, n$ & $10(43 \%)$ & $7(64 \%)$ & 0.47 \\
\hline Gender, female, $\mathrm{n}$ & $1(4 \%)$ & $3(27 \%)$ & 0.09 \\
\hline $\mathrm{BMI} \geq 22^{2}, \mathrm{n}$ & $12(52 \%)$ & $5(45 \%)$ & 1.00 \\
\hline Daily drinker, n & $11(48 \%)$ & $5(45 \%)$ & 1.00 \\
\hline Current smoker, $\mathrm{n}$ & $3(13 \%)$ & $5(45 \%)$ & 0.08 \\
\hline Experience in endoscopy for more than 5 times, $n$ & $18(78 \%)$ & $6(55 \%)$ & 0.23 \\
\hline \multicolumn{4}{|c|}{$\begin{array}{l}\text { Score } 2 \text {, completely seen; Score } 1 \text {, partially seen; Score } 0 \text {, not seen } \\
\text { BMI, body mass index } \\
{ }^{1} \text { Fisher's exact tests } \\
{ }^{2} \text { Median value of all the patients }\end{array}$} \\
\hline
\end{tabular}

\section{Factors associated with the incomplete visualization}

Association between patient characteristics and the observation scores are shown in $>$ Table 4 . Although it was not statistically significant, female gender $(P=0.09)$ and current smoking habit $(P=0.08)$ had a tendency toward the incomplete visualization.

\section{Discussion}

In this randomized, controlled, crossover trial, we demonstrated that pharyngeal examinations using the dedicated mouthpiece had a substantially higher rate of complete visualization of the hypopharynx than those using a conventional mouthpiece ( $68 \%$ vs. $0 \%$ ). The dedicated mouthpiece did not impair observation quality in the oropharynx or increase the incidence of adverse events compared with a conventional mouthpiece. This was the first study to directly compare this new method of pharyngeal observation with a conventional method, and clearly reveal its benefit.

A recent meta-analysis reported that prevalence of $\mathrm{H} \& \mathrm{~N}$ second primary cancers in patients with primary esophageal SCCS was $6.7 \%$; most of these (60\%) were located in the hypopharynx [23]. Moreover, a recent population-based study from the Netherlands indicated that a huge proportion of the hypopharynx cancer (68\%) are diagnosed in stage IV [24]. Thus, in high- risk patients of this kind, clinicians should thoroughly inspect the hypopharynx using the Valsalva maneuver. Taking into account the risk of hypopharyngeal cancer, patients with previously treated H\&N cancer [13], or habitual drinkers who have a flushing response to alcohol [25] could be the potential beneficiaries of the hypopharynx examination using the dedicated mouthpiece. In contrast, pharyngeal cancers are scarcely detected in patients without any history of cancer in the esophagus or $\mathrm{H} \& \mathrm{~N}$ region $(0.11 \% ; 10 / 8872)$ [13]. Considering that the dedicated mouthpiece is rather expensive, the Valsalva maneuver is not recommended in this low-risk population.

Success in the Valsalva maneuver requires pre-procedural instruction and cooperation from patients. In our practice, we always explain to patients why they must undergo detailed pharyngeal observation and instruct them in how to conduct the Valsalva maneuver. Before the actual examination, we ask them to bite the dedicated mouthpiece while blocking its opening with a finger. We then request that they forcefully exhale against the closed airway. If air comes out strongly from the opening upon release of the blocking finger, the Valsalva maneuver is being correctly performed [18].

Several techniques for performing the Valsalva maneuver during transoral endoscopy have been reported. Yamasaki et al. reported the "without-a-mouthpiece" Valsalva maneuver method, which requires no special device [26]. The drawback of this method is a risk of making bite damage to the endo- 
scope, which may cost much to repair. Iwatsubo et al. reported another Valsalva maneuver method using a pediatric mouthpiece, which is cheaper than the dedicated mouthpiece used in the present study [27]. However, the unusual placement of the entire mouthpiece into the mouth may be puzzling to the patients, and it risks unintentional swallowing. The rate of complete visualization of the hypopharynx was $76 \%$ in the Iwatsubo et al. study, which was higher than our result (68\%). However, the possibility of investigator bias should be taken into account, as their evaluation was carried out by the examiners themselves. Hence, there might be little difference in the capability of the complete hypopharyngeal visualization between their and our methods. In this context, the dedicated mouthpiece used in the current study would be an optimal option for the Valsalva maneuver during transoral endoscopy.

The current study had several limitations. First, we did not compare actual detection rates for hypopharyngeal cancer between the two observation methods. A large-scale, prospective study is required to elucidate whether the Valsalva maneuver improves detection of cancer. Second, we did not assess patients' degree of discomfort during the examinations; however, as no one asked to interrupt the examination, we speculate that the procedure was at least endurable. Third, because there was no gold standard of quality for hypopharyngeal visibility, we evaluated it with our original scoring criteria, which may have had inherent subjectivity. However, considering that interobserver agreements of the scoring among the three evaluators were high (pairwise kappa values of $0.84,0.68$, and 0.71 ), which indicates high reproducibility of our criteria, we believe our scoring system had considerable validity. Meanwhile, we also realize the necessity of further validating our scoring criteria; interobserver agreements should be assessed among a larger number of observers.

\section{Conclusion}

In conclusion, endoscopic view of the hypopharynx was significantly improved by the Valsalva maneuver using the dedicated mouthpiece compared to that of a conventional pharyngeal observation. We recommend including this new technique as a routine diagnostic procedure in patients with esophageal cancers, who are at high risk of developing second primary pharyngeal cancers.

\section{Acknowledgements}

The authors thank Editage (www.editage.com) for English language editing.

\section{Competing interests}

The authors declare that they have no conflict of interest.

\section{References}

[1] Slaughter DP, Southwick HW, Smejkal W. Field cancerization in oral stratified squamous epithelium: clinical implication of multicentric origin. Cancer 1953; 6: 963-968

[2] Katada C, Yokoyama T, Yano T et al. Alcohol consumption and multiple dysplastic lesions increase risk of squamous cell carcinoma in the esophagus, head, and neck. Gastroenterology 2016; 151: 860-869

[3] Katada C, Okamoto T, Kano K. Endoscopic diagnosis and treatment of superficial pharyngeal cancer. Dig Endosc 2017; 29: 824

[4] Shimizu Y, Yamamoto J, Kato M et al. Endoscopic submucosal dissection for treatment of early stage hypopharyngeal carcinoma. Gastrointest Endosc 2006; 64: 255-259

[5] Muto M, Satake H, Yano T et al. Long-term outcome of transoral organ-preserving pharyngeal endoscopic resection for superficial pharyngeal cancer. Gastrointest Endosc 2011; 74: 477-484

[6] lizuka T, Kikuchi D, Hoteya S et al. A new technique for pharyngeal endoscopic submucosal dissection: peroral countertraction (with video). Gastrointest Endosc 2012; 76: 1034-1038

[7] Hanaoka N, Ishihara R, Takeuchi Y et al. Endoscopic submucosal dissection as minimally invasive treatment for superficial pharyngeal cancer: phase II study (with video). Gastrointest Endosc 2015; 82: 1002-1008

[8] Satake H, Yano T, Muto M et al. Clinical outcome after endoscopic resection for superficial pharyngeal squamous cell carcinoma invading the subepithelial layer. Endoscopy 2015; 47: 11-18

[9] Muto M, Minashi K, Yano T et al. Early detection of superficial squamous cell carcinoma in the head and neck region and esophagus by narrow band imaging: A multicenter randomized controlled trial. J Clin Oncol 2010; 28: 1566-1572

[10] Katada C, Tanabe S, Koizumi W et al. Narrow band imaging for detecting superficial squamous cell carcinoma of the head and neck in patients with esophageal cell carcinoma. Endoscopy 2010; 42: 185190

[11] Nonaka S, Saito Y, Oda I et al. Narrow-band imaging endoscopy with magnification is useful for detecting metachronous superficial pharyngeal cancer in patients with esophageal squamous cell carcinoma. J Gastroenterol Hepatol 2010; 25: 264-269

[12] Goda K, Dobashi A, Tajiri H. Perspectives on narrow-band imaging endoscopy for superficial squamous neoplasms of the orohypopharynx and esophagus. Dig Endosc 2014; 26: (Suppl. 01): 1-11

[13] Nakanishi H, Doyama H, Takemura K et al. Detection of pharyngeal cancer in the overall population undergoing upper $\mathrm{Gl}$ endoscopy by using narrow-band imaging: A single-center experience, 2009-2012. Gastrointest Endosc 2014; 79: 558-564

[14] Morimoto H, Yano T, Yoda Y et al. Clinical impact of surveillance for head and neck cancer in patients with esophageal squamous cell carcinoma. World J Gastroenterol 2017; 23: 1051-1058

[15] Kato M, Ishihara R, Hamada K et al. Endoscopic surveillance of head and neck cancer in patients with esophageal squamous cell carcinoma. Endosc Int Open 2016; 4: E752-E755

[16] Spraggs PD, Harries ML. The modified Valsalva manoeuvre to improve visualization of the hypopharynx during flexible nasopharyngoscopy. J Laryngol Otol 1995; 109: 863-864

[17] Hamada K, Ishihara R, Yamasaki Y et al. Transoral endoscopic examination of head and neck region. Dig Endosc 2018; 30: 516-521

[18] Kato M, Hayashi Y, Takehara T. Valsalva maneuver to visualize the closed hypopharyngeal space during transoral endoscopy using a novel dedicated mouthpiece. Dig Endosc 2019; 31: e24-e25

[19] Duane TD. Valsalva hemorrhagic retinopathy. Trans Am Ophthalmol Soc 1972; 70: 298-313 
[20] Yamasaki Y, Ishihara R, Hanaoka $\mathrm{N}$ et al. Pethidine hydrochloride is a better sedation method for pharyngeal observation by transoral endoscopy compared with no sedation and midazolam. Dig Endosc 2017; $29: 39-48$

[21] Tsuji K, Doyama H, Takeda Y et al. Use of transoral endoscopy for pharyngeal examination: cross-sectional analysis. Dig Endosc 2014; 26: 344-349

[22] Faul F, Erdfelder E, Lang AG et al. G*Power 3: A flexible statistical power analysis program for the social, behavioral, and biomedical sciences. Behav Res Methods 2007; 39: 175-191

[23] van den Ven SEM, Bugter O, Hardillo JA et al. Screening for head and neck second primary tumors in patients with esophageal squamous cell cancer: A systematic review and meta-analysis. United European Gastroenterol J 2019; 7: 1304-1311
[24] Petersen JF, Timmermans AJ, van Dijk BAC et al. Trends in treatment, incidence and survival hypopharynx cancer: a 20 -year poplationbased study in the Netherlands. Eur Arch Otorhinolaryngol 2018; 275: 181-189

[25] Asakage T, Yokoyama A, Haneda T et al. Genetic polymorphisms of alcohol and aldehyde dehydrogenases, and drinking, smoking and diet in Japanese men with oral and pharyngeal squamous cell carcinoma. Carcinogenesis 2007; 28: 865-874

[26] Yamasaki Y, Ishihara R, Hamada K. Usefulness of the Valsalva maneuver without a mouthpiece to observe the hypopharynx using transoral endoscopy. Dig Endosc 2017; 29: 643-644

[27] Iwatsubo T, Ishihara R, Nakagawa K et al. Pharyngeal observation via transoral endoscopy using a lip cover-type mouthpiece. J Gastroenterol Hepatol 2019; 34: 1384-1389 